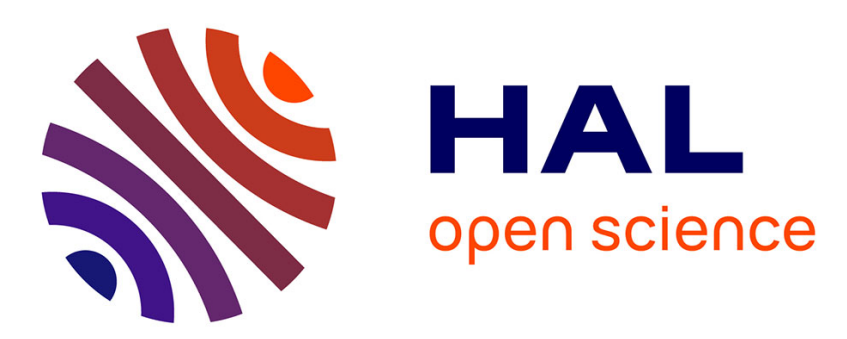

\title{
Interaction entre les dislocations extrinsèques du joint de grains et l'impureté carbone dans le fer $\alpha$ et ses alliages cubiques centrés
}

\author{
O. Khalfallah, L. Priester
}

\section{- To cite this version:}

O. Khalfallah, L. Priester. Interaction entre les dislocations extrinsèques du joint de grains et l'impureté carbone dans le fer $\alpha$ et ses alliages cubiques centrés. Revue de Physique Appliquée, 1981, 16 (4), pp.153-163. 10.1051/rphysap:01981001604015300 . jpa-00244908

\author{
HAL Id: jpa-00244908 \\ https://hal.science/jpa-00244908
}

Submitted on 1 Jan 1981

HAL is a multi-disciplinary open access archive for the deposit and dissemination of scientific research documents, whether they are published or not. The documents may come from teaching and research institutions in France or abroad, or from public or private research centers.
L'archive ouverte pluridisciplinaire HAL, est destinée au dépôt et à la diffusion de documents scientifiques de niveau recherche, publiés ou non, émanant des établissements d'enseignement et de recherche français ou étrangers, des laboratoires publics ou privés. 


\title{
Interaction entre les dislocations extrinsèques du joint de grains et l'impureté carbone dans le fer $\alpha$ et ses alliages cubiques centrés
}

\author{
O. Khalfallah et L. Priester \\ Laboratoire de Métallurgie Physique $(*)$, \\ Université Paris-Sud, 91405 Orsay Cedex, France \\ (Reçu le 19 novembre 1980, révisé le 8 janvier 1981, accepté le 12 janvier 1981)
}

\begin{abstract}
Résumé. - La stabilisation des dislocations extrinsèques des joints de grains du fer, des alliages fer- $4 \%$ nickel et fer-9\% chrome a été étudiée par microscopie électronique par transmission en fonction :

- de la géométrie du joint de grains (désorientation entre cristaux),

- de la présence ou non d'une ségrégation intergranulaire de carbone.

Le formalisme géométrique de Bollmann est opérant pour comprendre la présence de dislocations extrinsèques dans les joints spéciaux et leur élimination dans les joints généraux en l'absence d'impureté ségrégée.

En revanche, si une ségrégation active intervient, la différence de comportement des deux types de joints disparait, en particulier les joints généraux, nombreux dans les polycristaux réels, peuvent retenir des dislocations extrinsèques.
\end{abstract}

\begin{abstract}
The stabilization of extrinsic grain boundary dislocations in iron, iron-9 $\%$ chromium and iron-4 \% nickel alloys has been studied by transmission electron microscopy in relation with :

- the geometry of the grain boundary (disorientation between adjoining crystals),

- the possible occurrence of an intergranular segregation of carbon.

The geometrical approach of Bollmann cas satisfactorely explain the presence of extrinsic dislocation in special boundaries and their elimination in general boundaries if there are no segregated species in these boundaries. On the contrary, the occurrence of an active segregation involves that both type of boundaries have the same behaviour ; particularly, the general boundaries, in relatively high proportion in real polycrystals, can contain extrinsic dislocations.
\end{abstract}

1. Introduction. - Les dislocations extrinsèques résultent de l'interaction des dislocations de matrice avec la structure d'équilibre ou intrinsèque du joint de grains [1]. Leur formation et leurs réactions (accommodation-annihilation) contrôlent de nombreux processus mettant en jeu les interfaces, en particulier la déformation plastique et la recristallisation. Ces réactions sont complexes et dépendent :

- du mode de création des dislocations extrinsèques lors d'une déformation plastique à température ambiante ou lors de la croissance des grains;

- de la température de la réaction;

- du type de joint, spécial ou général [1];

- du type de dislocations extrinsèques : dislocations Vis glissiles dans l'interface ou dislocations Coin migrant par un processus de montée.

(*) Laboratoire associé au C.N.R.S., La 177.
Ces facteurs étant interdépendants, le comportement des dislocations extrinsèques reste mal établi.

Des travaux antérieurs $[2,3,4,5]$ ont différencié cependant le comportement des dislocations extrinsèques dans les joints spéciaux où elles seraient stabilisées, de leur comportement dans les joints généraux où un processus de dispersion interviendrait. Pumphrey et Gleiter [2] donnent deux interprétations possibles du phénomène de dispersion :

- dissociation en un grand nombre de dislocations de petits vecteurs de Burgers,

- élargissement du coeur de la dislocation.

Formellement, les deux mécanismes peuvent être considérés comme équivalents. La dispersion entraîne un réarrangement de la structure intrinsèque du joint de grains, elle nécessite une mobilité des atomes dans l'interface, elle est donc contrôlée par la diffusion intergranulaire [2]. 
Le rôle des impuretés comme frein à cette réaction est souvent évoqué, mais ne donne lieu à aucune étude systématique.

Notre objectif est d'étudier les conditions de stabilisation des dislocations extrinsèques en tentant de différencier l'influence des paramètres suivants :

- géométrie du joint de grains : désorientation entre cristaux adjacents;

- présence d'impuretés ségrégées dans l'interface.

2. Matériaux et méthodes. - Les matériaux de haute pureté utilisés sont le fer et les alliages $\mathrm{Fe}-9 \% \mathrm{Cr}$ et $\mathrm{Fe}-4 \% \mathrm{Ni}$ de structure cubique centrée; ils contiennent uniquement l'impureté carbone à de très faibles teneurs variant de $90 \times 10^{-6}$ à $250 \times 10^{-6}$ en poids.

Les échantillons sont soumis à divers traitements thermomécaniques de mise en forme : les plaquettes obtenues, d'épaisseur $1 / 10^{\mathrm{e}} \mathrm{mm}$ sont soumises à un recuit final de recristallisation de $24 \mathrm{~h}$ à une température élevée en domaine $\alpha$.

L'étude est effectuée en microscopie électronique par transmission à $100 \mathrm{kV}$ à l'aide d'un porte-objet basculement-rotation sur des lames minces préparées par la technique de la fenêtre. Elle est divisée en quatre étapes :

- détermination d'une relation d'orientation entre cristaux adjacents au joint;

- normalisation de la description du joint ;

- classification du joint en joint spécial et joint général;

- détermination de la direction des lignes de dislocation.

L'orientation de chaque grain dans le référentiel du microscope est obtenue en repérant trois pôles de plans non en zone dans des conditions de Laüe symétrique, qu'on reporte sur une projection stéréographique au 1/2 degré. A partir des pôles de trois paires de plans homologues dans les deux cristaux, on peut obtenir l'orientation relative de ces deux cristaux sous forme d'une rotation d'axe $R$ et d'angle $\theta$ (cf. Fig. 1). Compte tenu du ferromagnétisme du matériau, la précision sur l'angle est estimée à $\pm 2^{\circ}$.

Dans le système cubique, l'orientation relative de deux cristaux peut être décrite par 1124 rotations équivalentes. Il est donc nécessaire de normaliser la description du joint en utilisant la méthode des quaternions unitaires mise au point par Grimmer [6] pour les systèmes cubiques et étendue par Bonnet [7] aux autres systèmes cristallins. Cette normalisation permet de décrire la relation d'orientation entre grains en termes de DÉSORIENTATION qui est la rotation d'angle minimal et d'axe $[u, v, w]$, tels que $u \geqslant v \geqslant w \geqslant 0$. Cette désorientation est unique.

Les désorientations obtenues sont comparées aux désorientations de coïncidence dont les tables ont été publiées par différents auteurs $[8,9]$. Un joint est spécial C.S.L. (Coincidence Site Lattice) $[8,10,11]$

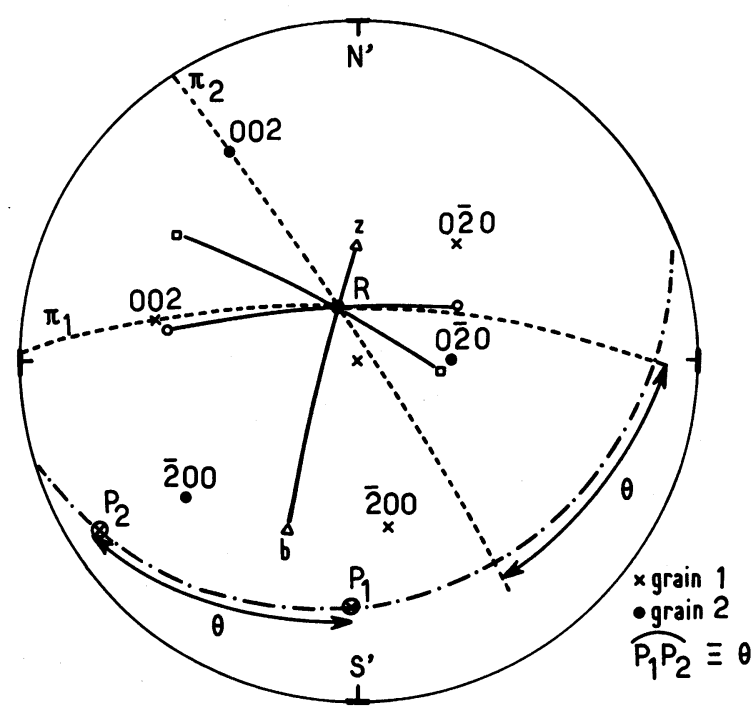

Fig. 1. - Détermination d'une relation d'orientation relative entre deux cristaux définie par un axe de rotation $R$ et un angle de rotation $\theta$ :

- l'arc $z b$ est la trace du plan bissecteur du dièdre formé par les plans (200) des deux cristaux,

- l'axe de rotation $R$ est obtenu à l'intersection de deux plans bissecteurs analogues au précédent,

- l'angle $\theta$ est mesuré entre les pôles $P_{1}$ et $P_{2}$ des plans homologues $\pi_{1}$ et $\pi_{2} ; \pi_{1}$ contient l'axe $R$ et un pôle d'indices simples $(h k l)_{1}$, $\pi_{2}$ contient l'axe $R$ et le pôle de mêmes indices $(h k l)_{2}$.

[Determination of a relative misorientation between two crystals by a rotation about an axis $R$, the rotation angle is $\theta$ :

- $z b$ is the trace of the bissecting plane of the-dihedral angle formed by the (200) planes of the crystals,

- the rotation axis $R$ is deduced by the intersection of another bissecting plane with the previous one,

- the angle of misorientation is measured between two poles $P_{1}$ and $P_{2}$ of similar planes $\pi_{1}$ and $\pi_{2} ; \pi_{1}$ contains $R$ and a low index pole $(k h l)_{1} ; \pi_{2}$ contains $R$ and the pole of crystal 2 with the same low index $(h k l)_{2}$.]

ou C.A.D. (Coincidence Axis Direction) [12] si sa désorientation est proche d'une position de coincidence. Un joint est général dans le cas contraire.

Les écarts admissibles à la coïncidence, définissant un critère de spécialité, ont été retenus après une analyse des travaux existants $[12,13]$ et en tenant compte de la précision de nos résultats (Tableau I).

Parallèlement à l'étude cristallographique des joints, la présence ou non de dislocations extrinsèques est notée et, le cas échéant, les directions de ces dislocations sont déterminées.

3. Résultats et interprétation. - 3.1 MORPHOLOGIE, GÉOMÉTRIE ET DISLOCATIONS EXTRINSÈQUES DES JOINTS DE GRAINS. - Nous avons étudié cinq matériaux :

fer-90 ppm C,

fer- $4 \% \mathrm{Ni}+90 \mathrm{ppm} \mathrm{C}$,

fer $-9 \% \mathrm{Cr}+90 \mathrm{ppm} \mathrm{C}$,

fer- $9 \% \mathrm{Cr}+150 \mathrm{ppm} \mathrm{C}$,

fer- $9 \% \mathrm{Cr}+250 \mathrm{ppm} \mathrm{C}$. 
Tableau I. - Ecarts angulaires $V$ à la coïncidence; $V$ : écart à la coïncidence; $V_{0}$ : limite angulaire des joints à faibles angles; $\Sigma$ : paramètre de la coincidence; $\Pi^{2}: h^{2}+k^{2}+l^{2}$ où $(h, k, l)$ sont les indices des plans en accord ; $a$ : paramètre de la maille cristalline; $b$ : module du vecteur de Burgers des dislocations de la matrice.

Modèle C.S.L.

$V=V_{0} . \Sigma^{-1 / 2}$ avec $V_{0}=8^{\circ}$

$\begin{array}{rll}\Sigma & & V \\ 1 & & - \\ 3 & & 4,6 \\ 5 & & 3,6 \\ 7 & & 3 \\ 9 & & 2,7 \\ 11 & & 2,4 \\ 13 & & 2,2 \\ 15 & & 2,1 \\ 17 & & 1,9 \\ 19 & & 1,8 \\ 21 & & 1,75 \\ 23 & & 1,7 \\ 25 & & 1,6\end{array}$

Le fer recristallisé présente une structure ferritique équilibrée (Fig. 2). Les lames minces montrent des joints de grains rectilignes, perpendiculaires aux faces de la lame (Fig. 3).

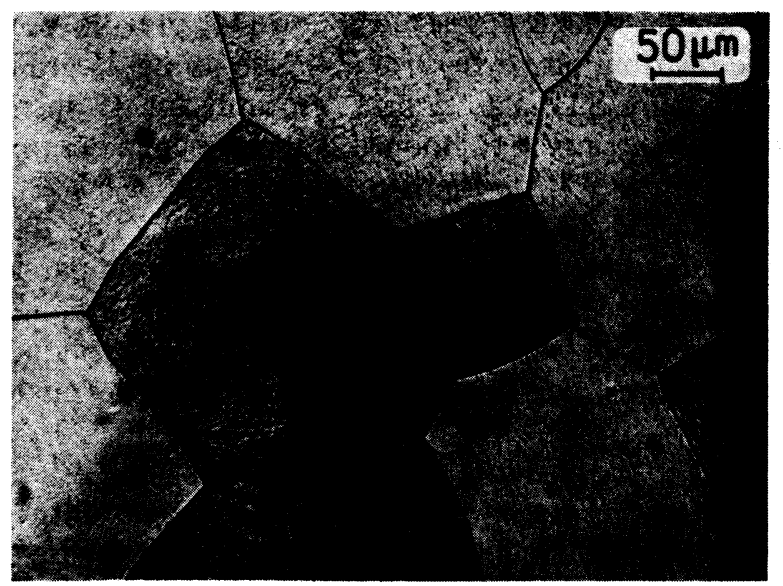

Fig. 2. - Ferrite équilibrée dans du fer contenant $90 \mathrm{ppm}$ de carbone recristallisé à $780^{\circ} \mathrm{C}$ pendant $24 \mathrm{~h}$.

[Equilibrated $\alpha$-iron containing $90 \mathrm{ppm}$ of carbon after recrystallisation at $780^{\circ} \mathrm{C}$ during $24 \mathrm{~h}$.]

L'alliage $\mathrm{Fe}-4 \% \mathrm{Ni}$ est recristallisé à grains fins irréguliers et à contours perturbés (Fig. 4). En microscopie électronique, ces joints paraissent sinueux, inclinés sur les faces de la lame et ayant une très faible densité de défauts extrinsèques (Fig. 5).

Après un maintien de $24 \mathrm{~h}$ à $780^{\circ} \mathrm{C}$, les structures des alliages $\mathrm{Fe}-9 \% \mathrm{Cr}$ ont des degrés de recristallisation variables selon la teneur en carbone.

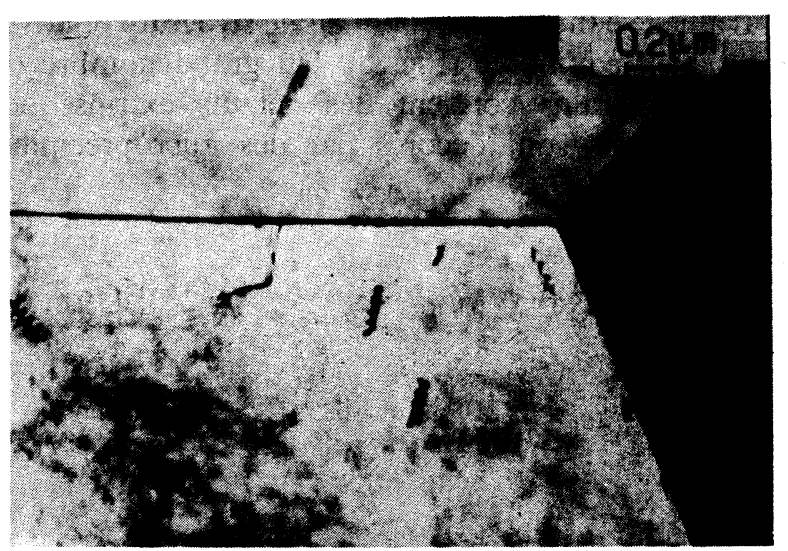

Fig. 3. - Aspects des joints de grains dans le fer, $90 \mathrm{ppm}$ de carbone, observés en microscopie électronique.

[Grain boundaries profiles in $\mathrm{Fe}, 90 \mathrm{ppm} \mathrm{C}$ observed by T.E.M.]

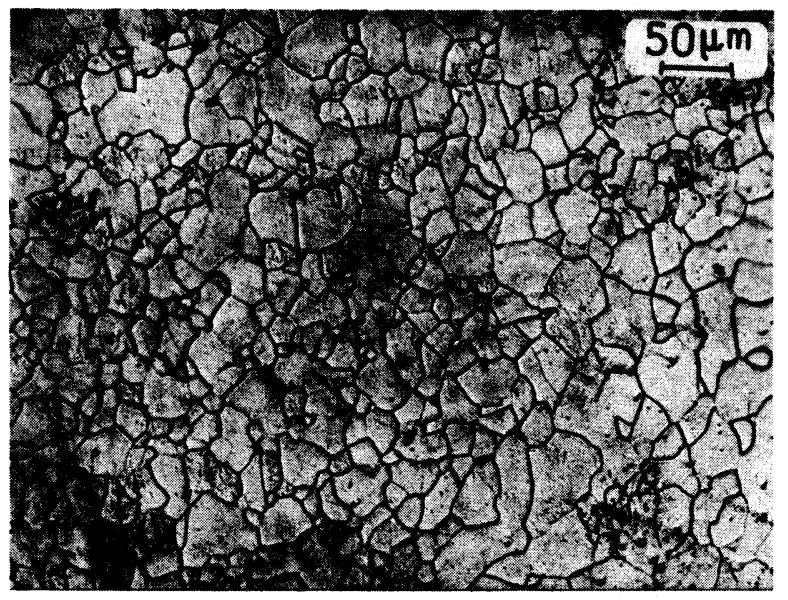

Fig. 4. - Ferrite perturbée dans un alliage fer-4\% nickel $(90 \mathrm{ppm}$ carbone) recristallisé à $550^{\circ} \mathrm{C}$ pendant $24 \mathrm{~h}$.

[Disturbed ferrite in $\mathrm{Fe}-4 \% \mathrm{Ni}(90 \mathrm{ppm} \mathrm{C})$ after recrystallisation at $550^{\circ} \mathrm{C}$ during $24 \mathrm{hrs}$.]

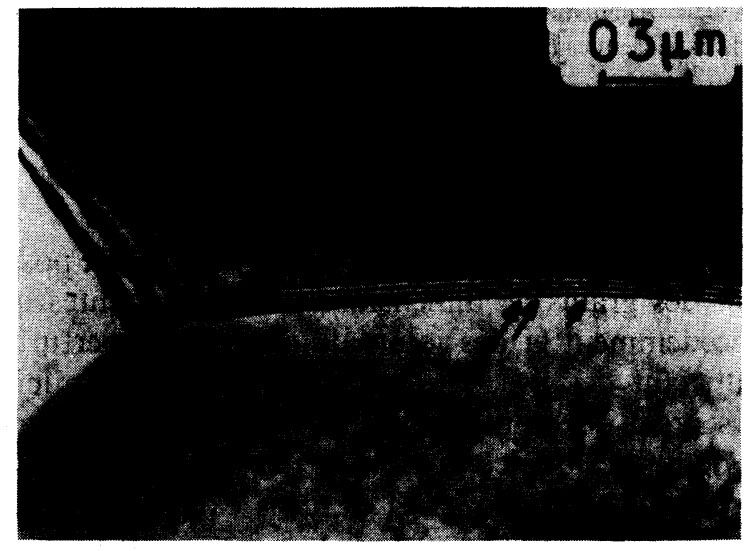

Fig. 5. - Micrographie électronique de joints de grains non équilibrés et contenant peu de défauts dans l'alliage fer $-4 \%$ nickel.

[Micrograph of no equilibrated grain boundaries with few defects in $\mathrm{Fe}-4 \% \mathrm{Ni}$.] 
L'alliage contenant $90 \mathrm{ppm}$ a une structure relativement bien équilibrée (Fig. 6). Un grand nombre de joints de grains contient des défauts extrinsèques dont certains sont décorés par des microprécipités (Fig. 7).

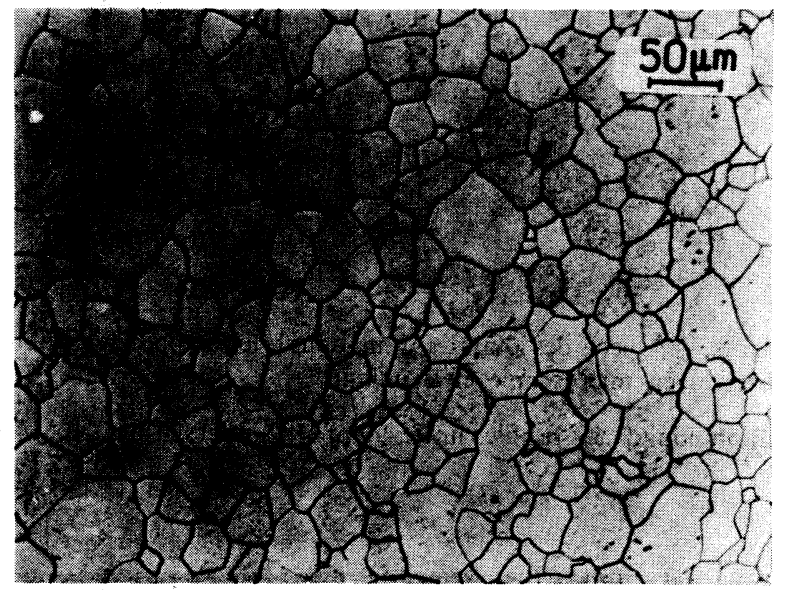

Fig. 6. - Microstructure de l'alliage fer-9 \% chrome (90 ppm carbone) après recristallisation à $780 \circ \mathrm{C}$ pendant $24 \mathrm{~h}$.

[Microstructure of $\mathrm{Fe}-9 \% \mathrm{Cr}(90 \mathrm{ppm} \mathrm{C})$ after recrystallisation at $780^{\circ} \mathrm{C}$ during $24 \mathrm{~h}$.]

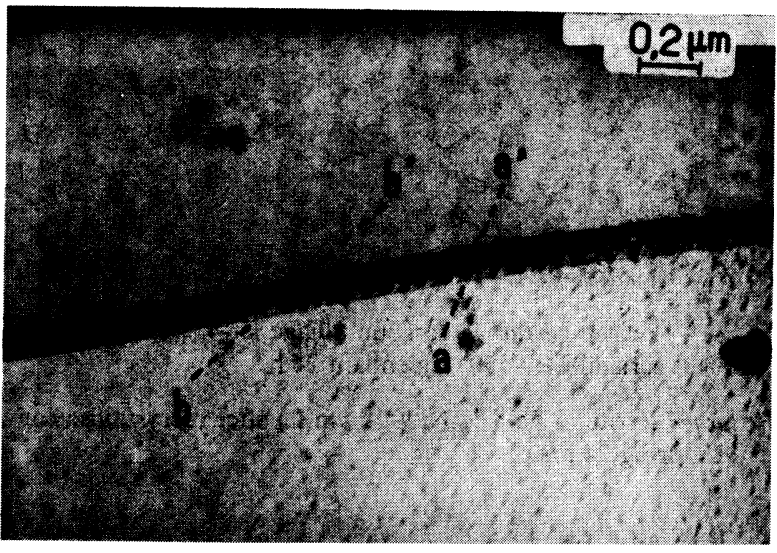

Fig. 7. - Alignement de microprécipités sur les lignes de dislocations extrinsèques $\left(\mathrm{aa}^{\prime}\right)$ et $\left(\mathrm{bb}^{\prime}\right)$.

[Micrograph of carbide precipitates which are decorating extrinsic dislocations lines in $\mathrm{aa}^{\prime}$ and $\mathrm{bb}^{\prime}$.]

Les alliages à $150 \mathrm{ppm}$ et $250 \mathrm{ppm}$ de carbone possèdent des grains de dimensions plus importantes que précédemment et des joints de grains plus perturbés contenant des précipités (Fig. 8). Les lames minces présentent des joints de grains très larges, rectilignes ou de formes quelconques avec des densités variables de défauts extrinsèques et de gros carbures du type $\mathrm{M}_{23} \mathrm{C}_{6}$ (Fig. 9). Aucune relation ne semble exister entre la présence de défauts et celle de précipités.

Notons que la plupart des observations effectuées aux différentes échelles sont cohérentes, en particulier pour l'état d'équilibre et la précipitation.

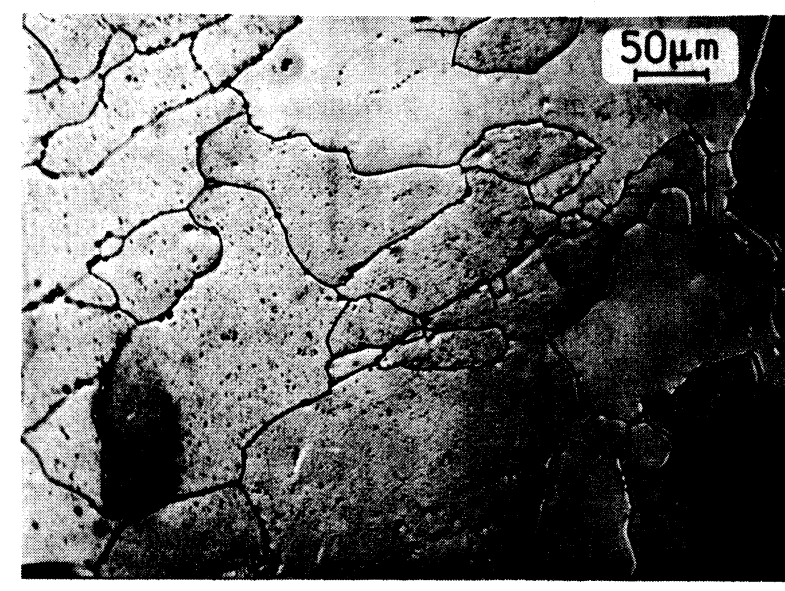

Fig. 8. - Observation en microscopie optique de l'alliage fer-9 $\%$ chrome contenant $250 \mathrm{ppm}$ de carbone, après recristallisation : les grains sont de taille plus importante que dans les nuances précédentes du même alliage - les précipités intergranulaires sont nombreux.

[Observation by T.E.M. of recrystallised $\mathrm{Fe}-9 \% \mathrm{Cr}$ alloy containing $250 \mathrm{ppm}$ of carbon : grain sizes are more important that previously and many intergranular precipitates are visible.]

Dans chaque matériau, nous avons considéré une quinzaine de joints de grains dont nous avons déterminé la désorientation (Tableaux II à VI).

Le nombre de joints spéciaux déterminé cristallographiquement, sensiblement $20 \%$ pour chacun des modèles C.S.L. et C.A.D. (cf. Tableaux II à VI), est à comparer aux résultats théoriques disponibles dans la littérature. Par exemple, Brandon [14] prévoit, pour une distribution au hasard, $42 \%$ de joints de grains du type C.S.L. Par contre, en utilisant le même critère Warrington et Boon [12] aboutissent à des proportions de $9 \%$ de joints du type C.S.L. et $60 \%$ du type C.A.D. Nos résultats, obtenus avec l'aide d'un critère géométrique semblable à ceux utilisés par les auteurs précédents, montrent des écarts par rapport aux prévisions théoriques qui peuvent s'expliquer, entre autres, par l'existence d'une texture des matériaux.

Parmi les résultats expérimentaux, signalons que quelques \% seulement de joints spéciaux ont été observés dans un alliage $\mathrm{Fe}-0,75 \%$ Mn sur la base de l'observation de réseaux de dislocations intrinsèques [15]. En tenant compte du critère de visualisation de la périodicité, la proportion de joints spéciaux dans nos échantillons tombe à $1 \%$ environ, en accord avec les travaux précédents.

On peut également remarquer que, malgré les traitements thermomécaniques équivalents, la quantité de joints spéciaux varie d'un matériau à l'autre. Dans le cas du fer (Tableau II), leur nombre, relativement élevé, est en accord avec l'observation en microscopie optique d'une structure bien équilibrée. L'absence de surconcentration en carbone dans les interfaces au cours de la recristallisation, permet aux joints de grains de migrer aisément et de tendre vers des positions énergétiquement favorables. 
a)

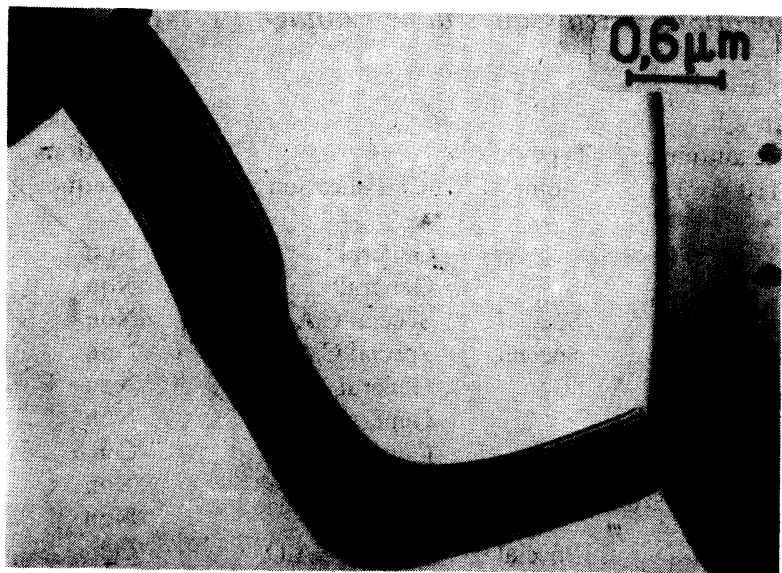

c)

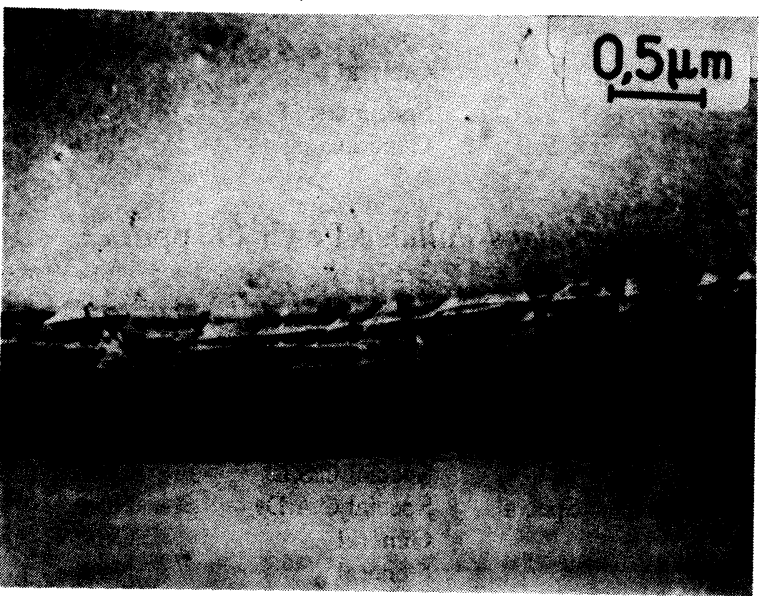

Fig. 9. - Divers aspects des joints de grains de l'alliage fer-9\% chrome ( $250 \mathrm{ppm}$ carbone) observés en microscopie électronique : a) joint sinueux contenant très peu de défauts,

b) présence d'un gros carbure maclé (type $\mathrm{M}_{23} \mathrm{C}_{6}$ dans un joint sans défaut),

c) nombreuses dislocations extrinsèques en l'absence de précipitation,

d) présence simultanée dans l'interface de défauts et de précipités.
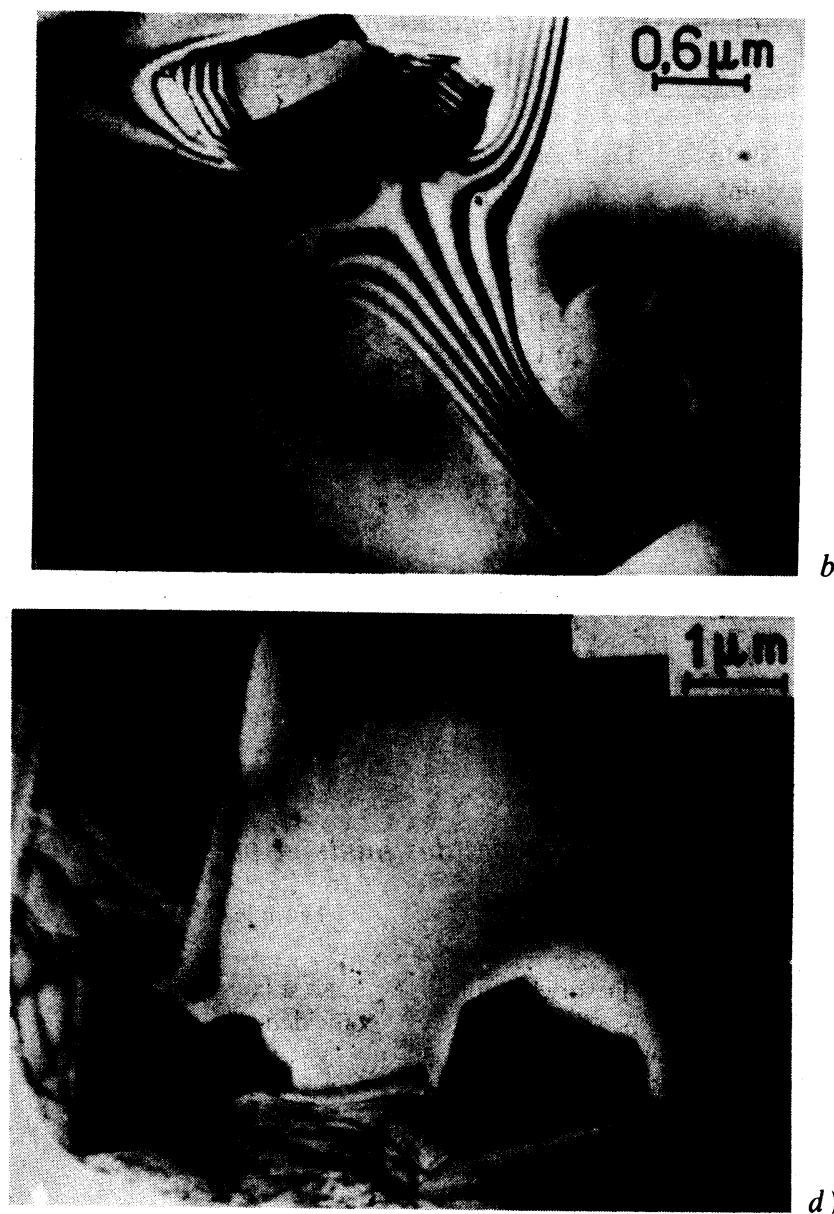

d)

[Different aspects of grain boundaries in $\mathrm{Fe}-9 \% \mathrm{Cr}(250 \mathrm{ppm} \mathrm{C})$ observed by T.E.M. :

a) curved boundary with few defects,

b) presence of a twinned carbide $\left(\mathrm{M}_{23} \mathrm{C}_{6}\right)$ in a grain boundary without defect,

c) many extrinsic dislocations without precipitation,

d) simultaneous occurence of defects and precipitates in the boundary.]

Tableau II. - Classification des joints de grains et présence de dislocations extrinsèques dans le fer.

\section{Modèle C.S.L.}

$\begin{array}{cc}\begin{array}{cc}\text { No du } \\ \text { joint }\end{array} & \begin{array}{c}\text { Désorientation } \\ \theta /[U V W]\end{array} \\ - & - \\ 1 & 61 / 332 \\ 2 & 49 / 332 \\ 3 & 4 / 551 \\ 4 & 40 / 310 \\ 5 & 38 / 111 \\ 6 & 47 / 100 \\ 7 & 39 / 111 \\ 8 & 48 / 210 \\ 9 & 24 / 111 \\ 10 & 26 / 111 \\ 11 & 53 / 221 \\ 12 & 52 / 211 \\ 13 & 56 / 332 \\ 14 & 58 / 331\end{array}$

Total :

\begin{tabular}{|c|c|}
\hline $\begin{array}{l}\text { Ecart à la } \\
\text { coïncidence } \\
\text { - }\end{array}$ & $\begin{array}{c}\text { Type du } \\
\text { joint } \\
-\end{array}$ \\
\hline${ }^{\circ} \operatorname{de} \Sigma=1$ & \\
\hline${ }^{\circ} \operatorname{de} \Sigma=7$ & Spécial \\
\hline $\begin{array}{l}{ }^{\circ} \operatorname{de} \Sigma=7 \\
{ }^{\circ} \operatorname{de} \Sigma=15\end{array}$ & $\begin{array}{l}\text { Spécial } \\
\text { Spécial }\end{array}$ \\
\hline${ }^{\mathrm{o}} \mathrm{de} \Sigma=13 \mathrm{~b}$ & Spécial \\
\hline
\end{tabular}

Modèle C.A.D.

Ecart à la

coincidence autour de Type du $\langle 100\rangle,\langle 110\rangle,\langle 111\rangle \quad$ joint

-

-

\section{Général}

Général

Spécial

Général

Spécial C.S.L.

Spécial C.A.D.

Spécial C.S.L.

Spécial C.S.L.

$2^{\circ}$ de $\Sigma=21$ a Spécial
Spécial C.A.D.

Spécial C.S.L.

Général

Général

Général

Général

Spéciaux :

Généraux :
Présence de dislocations extrinsèques

Non
Non
Non
Non
Oui
Non
Oui
Oui
Oui
Oui
Non
Non
Oui
Non
$5 / 7$
$1 / 7$


Tableau III. - Classification des joints et présence de dislocations extrinsèques dans l'alliage $\mathrm{Fe}-\mathrm{Ni}$.

\begin{tabular}{|c|c|c|c|c|c|c|c|}
\hline $\begin{array}{l}\text { No du } \\
\text { joint }\end{array}$ & $\begin{array}{c}\text { Désorientation } \\
\theta /[U V W]\end{array}$ & $\begin{array}{l}\text { Ecart à la } \\
\text { coïncidence }\end{array}$ & $\begin{array}{c}\text { Type du } \\
\text { joint }\end{array}$ & $\begin{array}{c}\text { Modèle C.A.D. } \\
\text { Ecart à la } \\
\text { coïncidence autour de } \\
\langle 100\rangle,\langle 110\rangle,\langle 111\rangle\end{array}$ & $\begin{array}{l}\text { Type du } \\
\text { joint }\end{array}$ & Classification & $\begin{array}{l}\text { Présence de } \\
\text { dislocations } \\
\text { extrinsèques }\end{array}$ \\
\hline - & - & - & - & - & - & - & - \\
\hline 1 & $47 / 221$ & & & & & Général & Non \\
\hline 2 & $42 / 430$ & & & & & Général & Non \\
\hline 3 & $43 / 100$ & & & $2^{\circ}$ de $\Sigma=29 \mathrm{a}$ & Spécial & Spécial C.A.D. & Non \\
\hline 4 & $20 / 111$ & & & $2^{\circ}$ de $\Sigma=21 \mathrm{a}$ & Spécial & Spécial C.A.D. & Non \\
\hline 5 & $13 / 211$ & & & & & Général & Non \\
\hline 6 & $59 / 322$ & & & & & Général & Non \\
\hline 7 & $11 / 210$ & & & & & Général & Oui \\
\hline 8 & $25 / 320$ & & & & & Général & Non \\
\hline 9 & $32 / 210$ & & & & & Général & Non \\
\hline 10 & $24 / 111$ & & & $2^{\circ} \mathrm{de} \Sigma=21 \mathrm{a}$ & Spécial & Spécial C.A.D. & Oui \\
\hline 11 & $12 / 321$ & & & & & Général & Non \\
\hline 12 & $14 / 421$ & & & & & Général & Non \\
\hline 13 & $2 / 321$ & $2^{\circ} \mathrm{de} \Sigma=1$ & & & & Spécial & Non \\
\hline 14 & $33 / 311$ & & & & & Général & Non \\
\hline Total : & & & & & & $\begin{array}{l}\text { Spéciaux : } \\
\text { Généraux : }\end{array}$ & $\begin{array}{l}1 / 4 \\
1 / 10\end{array}$ \\
\hline
\end{tabular}

Tableau IV. - Types des joints et présence de dislocations extrinsèques dans l'alliage $\mathrm{Fe}-\mathrm{Cr}(90 \mathrm{ppm} \mathrm{C})$.

Modèle C.S.L.

$\begin{array}{cc}\begin{array}{c}\text { No du } \\ \text { joint }\end{array} & \begin{array}{c}\text { Désorientation } \\ \theta /[U V W]\end{array} \\ - & - \\ 1 & 60 / 111 \\ 2 & 12 / 110 \\ 3 & 43 / 211 \\ 4 & 9 / 311 \\ 5 & 38 / 210 \\ 6 & 24 / 210 \\ 7 & 38 / 321 \\ 8 & 53 / 221 \\ 9 & 30 / 432 \\ 10 & 60 / 322 \\ 11 & 48 / 210 \\ 12 & 53 / 322 \\ 13 & 26 / 210 \\ \text { Total : } & \end{array}$

Modèle C.A.D.

Ecart à la

Ecart à la Type du coïncidence autour de Type du coïncidence joint $\langle 100\rangle,\langle 110\rangle,\langle 111\rangle$ joint

$2^{\circ}$ de $\bar{\Sigma}=3 \quad$ Spécial

$2^{\circ}$ de $\Sigma=15$

Spécial

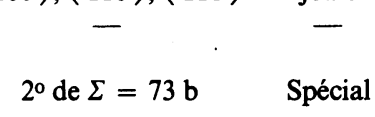

Classification

Spécial C.S.L.

Spécial C.A.D.

Général

Général

Général

Général

Général

Général

Général

Général

Spécial C.S.L.

Général

Général

Spéciaux :

Généraux :
Présence de dislocations extrinsèques

Oui

Non

Oui

Non

Non

Non

Non

Non

Oui

Non

Oui

Non

Oui

$2 / 3$

$3 / 10$

Tableau V. - Types des joints et présence de dislocations extrinsèques dans l'alliage $\mathrm{Fe}-\mathrm{Cr}(150 \mathrm{ppm} \mathrm{C})$.

Modèle C.S.L.

$\begin{array}{ccc}\begin{array}{cc}\text { No du } \\ \text { joint }\end{array} & \begin{array}{c}\text { Désorientation } \\ \theta /[U V W]\end{array} \\ - & & - \\ 1 & & 59 / 221 \\ 2 & & 47 / 310 \\ 3 & & 18 / 322 \\ 4 & & 47 / 322 \\ 5 & & 19 / 100 \\ 6 & & 60 / 111 \\ 7 & & 41 / 110 \\ 8 & & 39 / 320 \\ 9 & & 19 / 322 \\ 10 & 33 / 331 \\ 11 & 9 / 321 \\ 12 & 26 / 221 \\ 13 & 60 / 111 \\ 14 & 48 / 632 \\ \text { Total }: & \end{array}$

$2^{\circ}$ de $\Sigma=3$
Modèle C.A.D.

Ecart à la

Ecart à la Type du coïncidence autour de Type du

Spécial coïncidence joint

$\langle 100\rangle,\langle 110\rangle,\langle 111\rangle \quad$ joint

$2^{\circ}$ de $\Sigma=3 \quad$ Spécial

Spécial $2^{\circ}$ de $\Sigma=37$ a

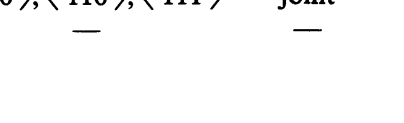

de $5=37$ a

\section{Classification}

Général

Général

Général

Général

Spécial C.A.D

Spécial C.S.L.

Spécial C.S.L.

Général

Général

Général

Général

Général

Spécial C.S.L.

Général

Spéciaux :

Généraux :
Présence de dislocations extrinsèques

Oui

Oui

Oui

Oui

Non

Non

Non

Non

Non

Oui

Non

Oui

Oui

Oui

$1 / 4$

$7 / 10$ 
Tableau VI. - Types des joints et présence de dislocations extrinsèques dans l'alliage $\mathrm{Fe}-\mathrm{Cr}$ (250 ppm C).

\begin{tabular}{|c|c|c|c|c|c|c|c|}
\hline \multirow[b]{2}{*}{$\begin{array}{l}\text { No du } \\
\text { joint }\end{array}$} & \multirow[b]{2}{*}{$\begin{array}{c}\text { Désorientation } \\
\theta /[U V W]\end{array}$} & \multicolumn{2}{|c|}{ Modèle C.S.L. } & \multicolumn{2}{|l|}{ Modèle C.A.D. } & & \multirow[b]{2}{*}{$\begin{array}{r}\text { Présence de } \\
\text { dislocations } \\
\text { extrinsèques }\end{array}$} \\
\hline & & $\begin{array}{l}\text { Ecart à la } \\
\text { coïncidence }\end{array}$ & $\begin{array}{c}\text { Type du } \\
\text { joint }\end{array}$ & $\begin{array}{c}\text { Ecart à la } \\
\text { coïncidence autour de } \\
\langle 100\rangle,\langle 110\rangle,\langle 111\rangle\end{array}$ & $\begin{array}{c}\text { Type du } \\
\text { joint }\end{array}$ & Classification & \\
\hline- & - & - & - & - & - & - & - \\
\hline 1 & $38 / 110$ & $2^{\circ}$ de $\Sigma=9$ & Spécial & & & Spécial C.S.L. & Non \\
\hline 2 & $10 / 111$ & & & $2^{\circ}$ de $\Sigma=73 \mathrm{a}$ & Spécial & Spécial C.A.D. & Non \\
\hline 3 & $50 / 110$ & $2^{\circ} \mathrm{de} \Sigma=11$ & Spécial & & & Spécial C.S.L. & Non \\
\hline 4 & $39 / 111$ & $2^{\circ}$ de $\Sigma=7$ & Spécial & & & Spécial C.S.L. & Non \\
\hline 5 & $55 / 111$ & $5^{\circ}$ de $\Sigma=3$ & & $2^{\circ} \mathrm{de} \Sigma=61 \mathrm{~d}$ & Spécial & Spécial C.A.D. & Non \\
\hline 6 & $49 / 111$ & $2^{\circ} \mathrm{de} \Sigma=19 \mathrm{~b}$ & & $2^{\circ} \mathrm{de} \Sigma=19 \mathrm{~b}$ & Spécial & Spécial C.A.D. & Non \\
\hline 7 & $2 / 321$ & $2^{\circ} \operatorname{de} \Sigma=1$ & & & & Spécial & Non \\
\hline 8 & $38 / 320$ & & & & & Général & Non \\
\hline 9 & $59 / 210$ & & & & & Général & Non \\
\hline 10 & $13 / 110$ & & & $3^{\circ}$ de $\Sigma=91 \mathrm{a}$ & Spécial & Spécial C.A.D. & Non \\
\hline 11 & $7 / 221$ & 70 de $\Sigma=1$ & & & & Spécial & Non \\
\hline 12 & $53 / 332$ & & & & & Général & Oui \\
\hline 13 & $9 / 210$ & & & & & Général & Oui \\
\hline Total & & & & & & $\begin{array}{l}\text { Spéciaux : } \\
\text { Généraux : }\end{array}$ & $\begin{array}{l}0 / 9 \\
2 / 4\end{array}$ \\
\hline
\end{tabular}

A teneur en carbone égale ( $90 \mathrm{ppm})$, l'adjonction de chrome ou de nickel tend à diminuer le nombre relatif de joints spéciaux (Tableaux III et IV); cette constatation est à rapprocher de la détection d'une ségrégation intergranulaire de carbone dans les deux alliages dès le début de la recristallisation $[16,17]$.

Le nombre élevé de joints spéciaux dans l'alliage $\mathrm{Fe}-9 \% \mathrm{Cr}(250 \mathrm{ppm})$ en contradiction avec la remarque précédente, peut être relié à la présence de nombreux précipités intergranulaires de taille importante. Ceux-ci constituent des puits de carbone, ils diminuent sa quantité en solution solide, en particulier dans les interfaces [18].

\subsection{Présence de dislocations eXtrinsèQues dans} LES JOINTS DE GRAINS. - Sur les tableaux II à VI, nous avons noté la présence ou l'absence de dislocations extrinsèques dans chaque joint que nous allons discuter en fonction des objectifs de l'étude.

3.2.1 Influence de la géométrie du joint de grains. Le tableau VII regroupe les résultats indépendemment de la nature de l'alliage pour dégager le rôle de la géométrie du joint seule. Il montre que les dislocations extrinsèques se répartissent avec la même pro- portion entre joints de grains spéciaux et généraux. Ce résultat est en contradiction avec le modèle de stabilisation basé uniquement sur la géométrie du joint $[3,5]$. En particulier, ce qui est important, c'est la présence de dislocations extrinsèques dans les joints de grains généraux, indiquant que le processus de dispersion de Pumphrey et Gleiter n'a pas eu lieu [2]. Ce processus étant activé thermiquement, on pourrait supposer que les dislocations extrinsèques ont été introduites par manipulation de la lame à la température ambiante; la considération des tableaux II à VI, montrant une répartition différente dans les deux types de joints de grains des matériaux pour des manipulations équivalentes des lames minces, tend à infirmer l'hypothèse précédente. Cependant, la possibilité de conserver des dislocations dans les joints de grains d'un matériau incomplètement recristallisé à haute température, est fortement controversée $[2,4]$; nous avons donc jugé nécessaire de vérifier le comportement des dislocations extrinsèques observées, lors d'un recuit de $30 \mathrm{~s}$ à $1 \mathrm{~min}$ à $600^{\circ} \mathrm{C}$, conditions pour lesquelles les phénomènes de dispersion des dislocations extrinsèques résultant d'une déformation plastique à température ambiante

Tableau VII. - Influence du type de joint sur la présence de dislocations extrinsèques.

\begin{tabular}{|c|c|c|c|c|c|}
\hline $\begin{array}{c}\text { Type } \\
\text { du joint }\end{array}$ & $\begin{array}{l}\text { Nombre } \\
\text { de joints } \\
\text { observés }\end{array}$ & $\begin{array}{c}\text { Proportion } \\
\%\end{array}$ & $\begin{array}{c}\text { Présence } \\
\text { de dislocations } \\
\text { extrinsèques }\end{array}$ & $\begin{array}{l}\text { Nombre } \\
\text { de joints }\end{array}$ & $\begin{array}{c}\text { Proportion } \\
\%\end{array}$ \\
\hline- & - & - & - & - & - \\
\hline Spécial & 27 & 40 & $\begin{array}{l}\text { Oui } \\
\text { Non }\end{array}$ & $\begin{array}{r}9 \\
18\end{array}$ & $\begin{array}{l}33 \\
67\end{array}$ \\
\hline Non spécial & 41 & 60 & $\begin{array}{l}\text { Oui } \\
\text { Non }\end{array}$ & $\begin{array}{l}14 \\
27\end{array}$ & $\begin{array}{l}34 \\
66\end{array}$ \\
\hline Total & 68 & 10 & $\begin{array}{l}\text { Oui } \\
\text { Non }\end{array}$ & $\begin{array}{l}23 \\
45\end{array}$ & $\begin{array}{l}34 \\
66\end{array}$ \\
\hline
\end{tabular}


ont normalement lieu. En particulier, rappelons que dans un alliage $\mathrm{Fe}-\mathrm{Mn}$, quelques secondes à $370^{\circ} \mathrm{C}$ suffisent à faire disparaître ces dislocations [15].

Les observations ont été effectuées sur lames double-jet dont l'épaisseur observable peut atteindre $1 \mu \mathrm{m}$; nous avons constaté deux types de comportement :

- au voisinage du bord de la lame, les joints se déplacent pour se mettre perpendiculaires aux faces de la lame et, ainsi, abaisser leur énergie de surface. Dans ce cas, nous n'observons plus de dislocations extrinsèques ;

- au-delà d'une certaine distance du bord, les joints fortement inclinés sur les faces de la lame sont quasi stables; la plupart des dislocations extrinsèques sont toujours visibles (Fig. 10a et $b$ ), même si quelques déplacements très localisés peuvent intervenir.

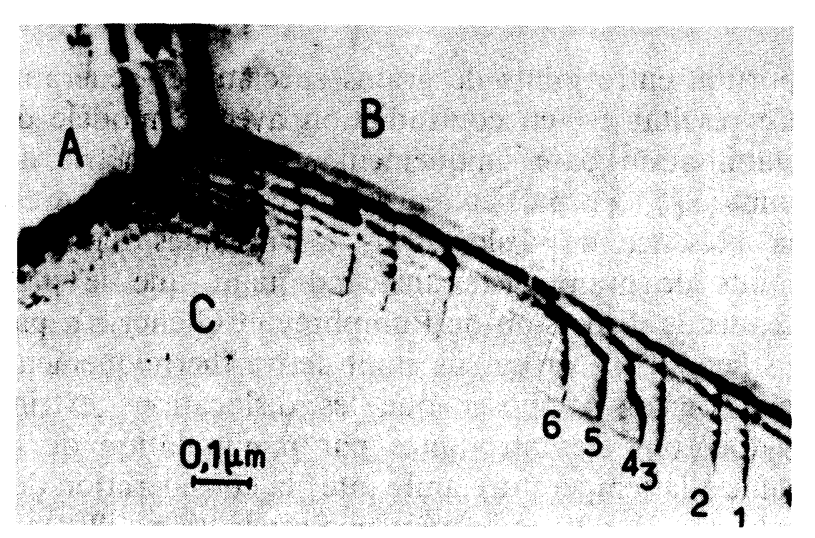

a)

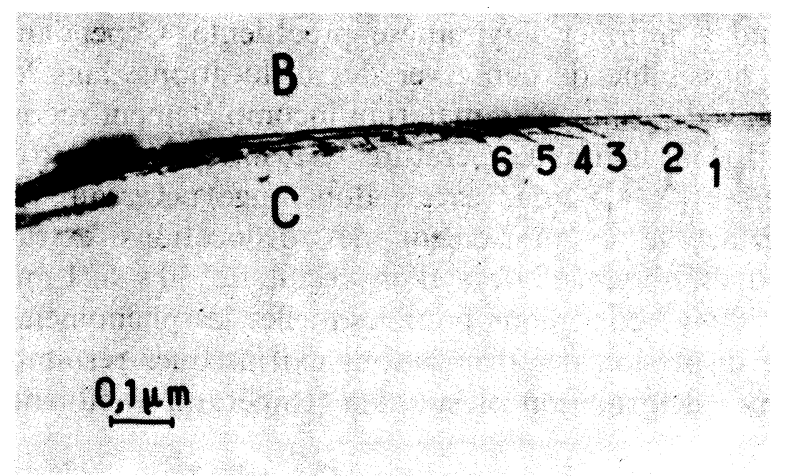

b)

Fig. 10. - Mise en évidence de la stabilité des dislocations extrinsèques du joint $\mathrm{B} / \mathrm{C}$ d'une lame mince double-jet :

a) observation de la lame mince obtenue à partir du matériau massif trempé depuis $780^{\circ} \mathrm{C}$,

b) observation de la lame mince après son maintien à $600^{\circ} \mathrm{C}$ pendant $1 \mathrm{~min}$.

[Stability of extrinsic grain boundary dislocations is observed in $\mathrm{B} / \mathrm{C}$ boundary of a double-jet thin foil :

a) observation of the thin foil providing from the bulk material quenched at $780^{\circ} \mathrm{C}$,

b) observation of the same thin foil after a following heat treatment at $600^{\circ} \mathrm{C}$ during $1 \mathrm{~min}$.]
L'expérience prouve que c'est seulement dans le cas où le joint migre - c'est-à-dire si quelques-uns des cinq degrés de liberté, sinon tous, du joint de grains changent (plan et désorientation ou plan seul) - que les dislocations extrinsèques peuvent s'annihiler. Au bord de la lame, le processus de dispersion intervient car les forces motrices de surface sont importantes, mais les comportements de ces joints ne sont pas représentatifs de ceux du matériau massif.

Dans les parties les plus épaisses, les joints sont dans un état métastable résultant de la recristallisation; les effets de surface sont insuffisants pour provoquer leur migration et leurs réactions sont caractéristiques du matériau massif. Rappelons, à l'appui de ceci que $D$. Bouchet, après une étude statistique, sans référence à la cristallographie des interfaces, continue à observer des dislocations extrinsèques dans les joints de grains d'alliages $\mathrm{Fe}-9 \% \mathrm{Cr}$, contenant sensiblement la même teneur en carbone, après des traitements de 22 jours à $780^{\circ} \mathrm{C}$; en fait, leur quantité évolue peu avec la durée du traitement au-delà de $24 \mathrm{~h}$ [19]. Nous pensons donc que la plupart des dislocations extrinsèques observées à température ambiante sur les lames classiques résultent de leur stabilisation à la température de $780^{\circ} \mathrm{C}$ intervenant lors de la croissance des grains et dépendant d'autres facteurs que la structure du joint de grains seule. En particulier la présence d'impuretés telles que le carbone peut avoir un rôle par analogie à celui des atmosphères de Cottrell dans la matrice, rôle évoqué par de nombreux auteurs $[2,5,20]$.

3.2.2 Influence des éléments ségrégés aux interfaces. - Nous avons analysé le comportement de

Tableau VIII. - Influence des ségrégations intergranulaires sur la stabilité des dislocations extrinsèques. $n$ : nombre de joints observés; $n_{\mathrm{s}}$ : nombre de joints spéciaux; $n_{\mathrm{g}}$ : nombre de joints généraux; $N_{\mathrm{s}}$ : nombre de joints spéciaux contenant des dislocations extrinsèques; $N_{\mathrm{g}}$ : nombre de joints généraux contenant des dislocations extrinsèques.

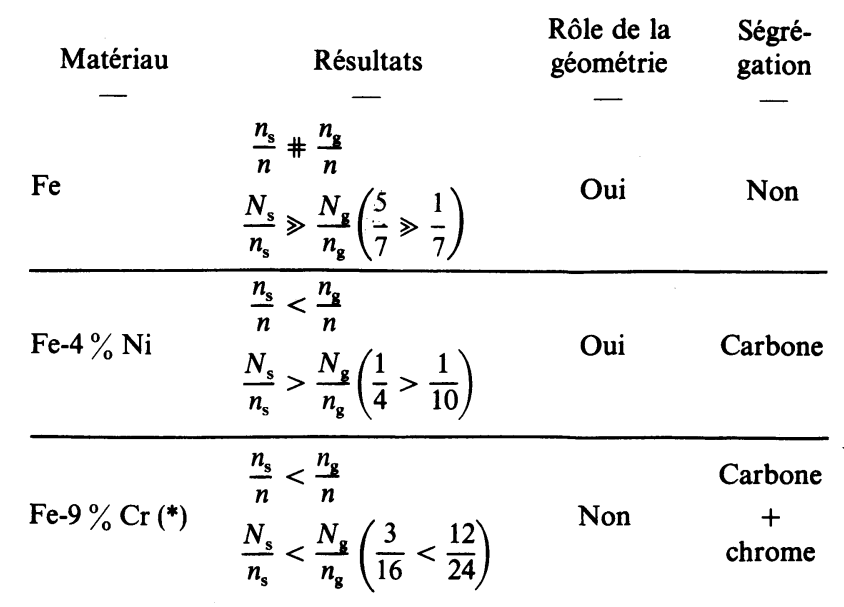

$\left(^{*}\right)$ Les 3 nuances de carbone sont prises en compte. 
chaque matériau en nous attachant aux phénomènes de ségrégation. Le tableau VIII résume les résultats.

Dans le cas $d u$ fer où il y a distribution homogène du carbone dans l'échantillon, comme l'ont montré des études d'autoradiographie à haut pouvoir de résolution du carbone 14 [16], la quantité de carbone dans les interfaces doit être insuffisante pour influer sur les processus de dispersion des dislocations de matrice. Celles-ci s'incorporent dans les joints généraux en fin de migration, d'où l'absence de dislocation extrinsèque dans ce type de joints. Leur présence dans les joints de grains spéciaux traduit le fait que parmi les réactions possibles de la dislocation de matrice avec le joint, la dispersion est peu probable [4] et la dissociation doit donner lieu à des dislocations extrinsèques dont les vecteurs $\left(b_{\mathrm{DSC}}\right.$ ) sont suffisamment importants pour qu'elles soient visibles en microscopie électronique par transmission [1].

Dans le cas des alliages $\mathrm{Fe}-9 \% \mathrm{Cr}$ et $\mathrm{Fe}-4 \% \mathrm{Ni}$ il y a ségrégation du carbone dans les interfaces des deux matériaux [16]. Cependant, le comportement des joints de grains généraux vis-à-vis de la stabilisation des dislocations extrinsèques diffère. Ces dislocations sont très peu nombreuses dans l'alliage fernickel, comme dans le fer, alors qu'elles ont tendance à être stabilisées dans l'alliage fer-chrome.

Le carbone, seul, ségrégé aux interfaces, n'est donc pas responsable des modifications des réactions de dislocations de matrice avec les joints de grains des alliages de fer. L'interprétation des résultats peut être recherchée dans la différence d'interaction du carbone avec l'élément d'alliage substitutionnel : répulsion vis-à-vis du nickel, attraction vis-à-vis du chrome. La ségrégation conjointe des éléments chrome et carbone dans les joints généraux de l'alliage $\mathrm{Fe}$ $9 \% \mathrm{Cr}$ peut être considérée comme une ségrégation active vis-à-vis de la stabilisation des dislocations extrinsèques. Ce résultat est en accord avec certains modèles théoriques, en effet l'association des deux paramètres, joint fortement désordonné et interaction électronique forte entre atomes ségrégés, conduit, selon Balluffi, à un cas extrême de ségrégation qui se manifeste par la présence d'un film continu bidimensionnel dans l'interface [21]. Cette répartition homogène des éléments ségrégés peut agir de deux façons sur le blocage des dislocations extrinsèques :

- soit elle favorise l'association des éléments ségrégés avec les dislocations qui s'incorporent dans le joint et qui constitueraient ultérieurement des sites favorables pour la nucléation des précipités [ 22 à 26];

- soit elle freine les mécanismes de diffusion intergranulaire, qui permettent d'interpréter le phénomène de dispersion [2] sans qu'il soit nécessaire d'envisager initialement une association étroite impuretédéfaut.

Dans un autre cas extrême, où le joint est spécial et où les éléments de l'alliage sont sans affinité pour les atomes d'impuretés, ceux-ci occupent des sites interstitiels ou vacants dans l'interface et peuvent diffuser d'un site à l'autre.

Dans un joint général, la répartition des atomes ségrégés doit dépendre de l'ampleur de la ségrégation; celle-ci est fonction de la quantité globale d'impureté dans la matrice mais aussi de l'interaction chimique de cette impureté avec les éléments d'alliage; en effet, selon Hondros, le coefficient d'enrichissement est inversement proportionnel à la limite de solubilité de l'impureté [27], elle-même fonction de l'affinité de celle-ci pour les éléments constitutifs du matériau.

La différence de concentration entre impuretés en solution solide dans les joints et dans la matrice est résorbée par la formation et la croissance des précipités intergranulaires [28]. Ceci peut expliquer les résultats obtenus sur l'alliage à $250 \mathrm{ppm}$ de carbone (Tableau VI) résultats qui apparaissent contradictoires si on tient uniquement compte de la quantité totale de carbone dans le matériau.

\subsubsection{Direction des lignes de dislocations extrin-} sèques. - Dans la plupart des cas nous observons dans les joints de grains un ensemble de dislocations orientées le long d'une direction privilégiée; nous déterminons cette direction en l'indexant dans cha-

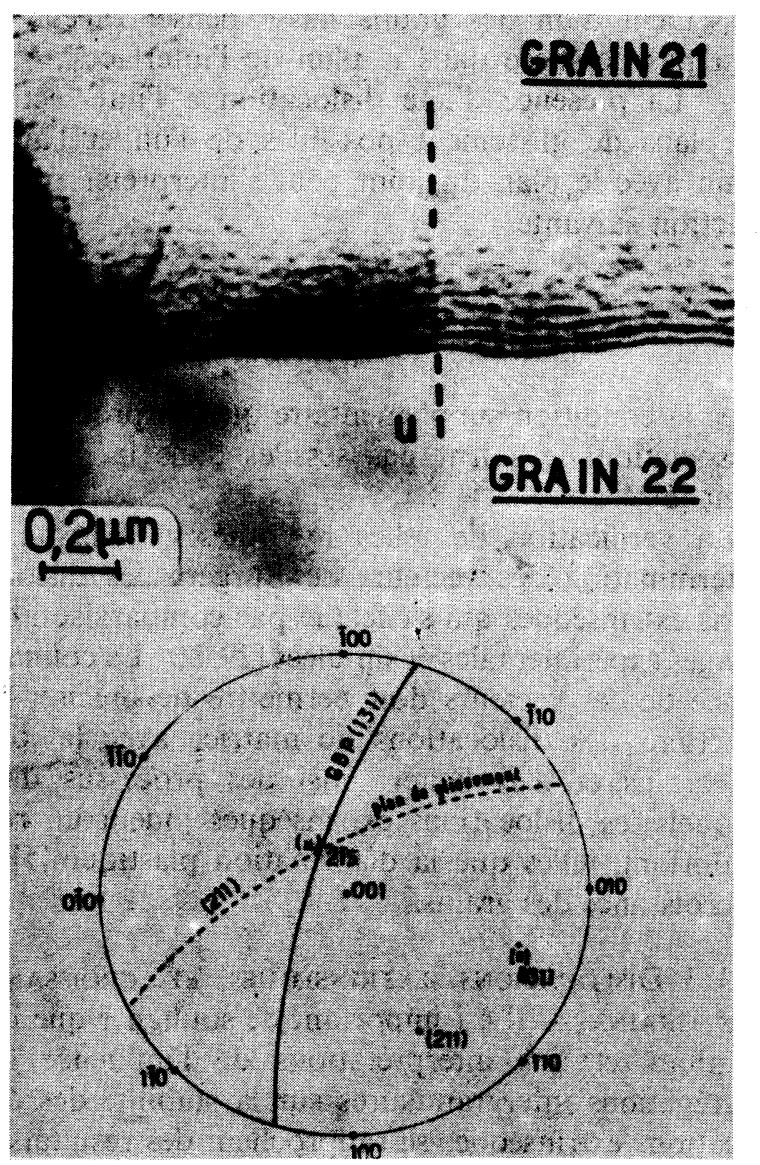

Fig. 11. - Indexation d'une ligne de dislocation dans un joint spécial : $u=[\overline{215}]=(211) \wedge(131)$.

[Indexation of a dislocation line in a special boundary :

$$
u=[\overline{215}]=(211) \wedge(131) .]
$$


Tableau IX. - Détermination de la direction de la ligne de dislocation.

\begin{tabular}{|c|c|c|c|c|c|c|c|c|}
\hline \multirow[b]{2}{*}{ Matériau } & \multirow[b]{2}{*}{$\begin{array}{l}\text { Orientation } \\
\text { relative } \\
\theta /[u, v, w]\end{array}$} & \multirow[b]{2}{*}{ Spécialité } & \multicolumn{3}{|c|}{ Grain supérieur } & \multicolumn{3}{|c|}{ Grain inférieur } \\
\hline & & & $\begin{array}{c}\text { Plan } \\
\text { du } \\
\text { joint }\end{array}$ & $\begin{array}{c}\text { Direction } \\
\text { de la ligne } \\
\text { de dislocation }\end{array}$ & $\begin{array}{l}\text { Plan de } \\
\text { glisse- } \\
\text { ment }\end{array}$ & $\begin{array}{c}\text { Plan } \\
\text { du } \\
\text { joint }\end{array}$ & $\begin{array}{c}\text { Direction } \\
\text { de la ligne } \\
\text { de dislocation }\end{array}$ & $\begin{array}{c}\text { Plan de } \\
\text { glisse- } \\
\text { ment }\end{array}$ \\
\hline $1 \mathrm{Fe}-\overline{9} \% \mathrm{Cr}$ & $17 \overline{\overline{1}} 12$ & $\overline{\text { Oui }}$ & $\overline{(164)}$ & {$[\overline{2 \overline{3}} 4]$} & $\overline{(121)}$ & $\overline{(124)}$ & {$[\overline{4443}]$} & $\overline{(110)}$ \\
\hline $2 \mathrm{Fe}-9 \% \mathrm{Cr}$ & $60 / \underline{012}$ & Oui & (133) & [312] & (110) & $(5 \overline{3} 3)$ & [372] & (112) \\
\hline $3 \mathrm{Fe}-9 \% \mathrm{Cr}$ & $134 / \overline{3} 25$ & Non & $(\underline{1} 00)$ & [001] & (110) & (112) & [311] & (121) \\
\hline $4 \mathrm{Fe}-9 \% \mathrm{Cr}$ & $151 / \overline{2} 13$ & Oui & $(\underline{1} 20)$ & {$[2 \overline{1} 3]$} & (211) & $(131)$ & {$[\overline{215}]$} & (211) \\
\hline $5 \mathrm{Fe}-9 \% \mathrm{Cr}$ & $26 / 120$ & Non & (116) & [331] & & $(\overline{2} 03)$ & [342] & $(\overline{2} 11)$ \\
\hline $6 \mathrm{Fe}-9 \% \mathrm{Cr}$ & $148 / \overline{2} 03$ & Oui & $(\overline{1} 12)$ & {$[1 \overline{3} 2]$} & & $(\overline{2} 43)$ & {$[1 \overline{1} 2]$} & (110) \\
\hline $7 \mathrm{Fe}-9 \% \mathrm{Cr}$ & $86 / \overline{123}$ & Oui & $(3 \overline{1} 1)$ & [114] & (110) & (131) & {$[\overline{3} 23]$} & \\
\hline
\end{tabular}

cun des grains adjacents (Fig. 11). Les joints de grains dans lesquels de telles analyses ont été effectuées sont caractérisés par l'orientation relative de deux grains et le plan du joint (Tableau IX).

Nous constatons que la direction de la ligne de dislocations est souvent l'intersection d'un plan de glissement de l'un ou des deux grains et du plan du joint :

$$
(h k l)_{\text {joint }} \wedge\left(h^{\prime} k^{\prime} l^{\prime}\right)_{\text {glissement }}=\mathbf{u} .
$$

- L'appartenance d'une dislocation à un plan de glissement d'un des grains laisse penser qu'elle ne s'est pas déplacée dans le plan de l'interface.

- La présence d'une dislocation à l'intersection de plans de glissement possibles de l'un et l'autre grain avec le plan du joint peut s'interpréter par la réaction suivante :

$$
\frac{a}{2}\langle 111\rangle_{\mathrm{A}} \rightarrow \frac{a}{2}\langle 111\rangle_{\mathrm{B}}+\mathrm{b}_{\mathrm{DSC}}
$$

avec la condition supplémentaire que les produits de dissociation ne soient pas sessiles dans le plan du joint [5].

$\mathrm{La}$ vérification de telles hypothèses nécessite la détermination des vecteurs de Burgers des dislocations extrinsèques qui s'effectue par comparaison des images expérimentales et simulées [29,30]. La connaissance de ces vecteurs doit permettre de préciser les réactions des dislocations de matrice avec le joint de grains et d'expliciter ainsi des processus dans lesquels les dislocations extrinsèques jouent un rôle important, telles que la déformation plastique [5] et la croissance des grains.

\subsection{Dislocations extrinsèQues et CROISSANCE} DES GRAINS. - Il est important de souligner que nos résultats et nos interprétations de l'influence des ségrégations intergranulaires sur la stabilité des dislocations extrinsèques se rapprochent des résultats et des théories concernant le rôle des impuretés sur la migration des joints de grains, en particulier lors de la croissance des grains après la recristallisation primaire lorsque la force motrice est due à l'énergie interfaciale.
Il est bien connu que quelques ppm d'éléments ajoutés dans un métal pur, et susceptibles de ségréger, sont capables de freiner la migration des interfaces avec un effet incomparablement plus important sur les joints généraux [31]; la vitesse de migration peut diminuer de plusieurs puissances de dix [32].

Selon Bauer, il est quasi impossible d'envisager la migration d'un joint en considérant une répartition uniforme des impuretés dans le plan du joint [33], ce qui est le cas dans l'hypothèse du film continu. Une précipitation intergranulaire entraînant des fluctuations de composition le long du joint de grains est alors favorable à la mobilité des segments de joints situés entre les précipités et appauvris en impuretés [18]. Dans le même ordre d'idées, signalons également que Boutin constate une augmentation de la vitesse de migration des joints résultant de la diminution de la quantité de fer en solution solide dans l'aluminium lors d'une précipitation [28].

Sur la base de ce parallélisme entre les effets d'une ségrégation intergranulaire sur la stabilité des dislocations extrinsèques d'une part, et sur la migration des interfaces d'autre part, nous allons tenter d'interpréter les phénomènes intervenant lors du traitement de recristallisation des alliages.

Lors de la recristallisation primaire, les joints migrent en drainant leurs impuretés [28, 34]; les dislocations de matrice s'incorporent et se dissocient et leurs produits s'annihilent rapidement dans l'interface car la force motrice, due à la différence de densité de dislocations dans les grains contigus est suffisamment importante pour éviter les effets d'ancrage. Si une précipitation intervient à ce stade, les segments entre les précipités se comportent comme précédemment et linterface prend une forme complexe.

Lors de la croissance des grains, les dislocations extrinsèques peuvent être bloquées par les impuretés quand la force motrice de migration, liée à une diminution de l'énergie de surface, n'est plus suffisante.

En l'absence de ségrégation, les processus de recristallisation ont lieu rapidement et conduisent à des microstructures équilibrées pour lesquelles l'énergie des joints de grains et leur comportement vis-à-vis des dislocations extrinsèques peuvent s'interpréter 
uniquement sur la base du modèle géométrique, ce qui est le cas du fer.

En présence d'une ségrégation, lors de la recristallisation primaire, la présence constante d'impuretés dans les joints de grains conduit à des positions d'énergie minimale différentes de celles prévues par le modèle géométrique ce qui peut expliquer le faible nombre de joints spéciaux dans les alliages.

Selon que la ségrégation est active ou non, la croissance des grains est plus ou moins freiné; en effet, dans l'alliage $\mathrm{Fe}-\mathrm{Ni}$ le carbone seul n'empêche pas la dispersion des dislocations extrinsèques dans les joints généraux alors que dans les alliages $\mathrm{Fe}-\mathrm{Cr}$, contenant 90 et $150 \mathrm{ppm}$ de carbone, son association avec le chrome gêne ce processus.

En présence d'une forte précipitation, ce qui est le cas de l'alliage $\mathrm{Fe}-\mathrm{Cr}$ à $250 \mathrm{ppm} \mathrm{C}$, les phénomènes sont plus complexes. Cependant, les schémas précédents peuvent être appliqués aux segments de joints libres de précipités.

Ces interprétations appuient l'hypothèse selon laquelle les réactions de formation et de dissociation des dislocations extrinsèques contrôlent les processus de croissance des grains. Rappelons qu'un même raisonnement a été développé par Dingley et Pond en ce qui concerne l'importance des dislocations extrinsèques dans la déformation plastique d'un matériau [5].
L'étude des paramètres pouvant influer sur la stabilité des dislocations extrinsèques est donc bien fondamentale pour comprendre les propriétés des interfaces.

4. Conclusion. - Le comportement des dislocations extrinsèques dans les joints de grains du fer, en l'absence de toute ségrégation, dépend essentiellement de critères géométriques : ces dislocations sont stables dans les joints spéciaux et s'annihilent par dispersion dans les joints généraux. Dans les joints de-grains des alliages où peuvent intervenir des ségrégations de l'impureté carbone et des éléments constitutifs de l'alliage, la nature, l'état et la répartition des espèces ségrégées influent sur la stabilité des dislocations; en particulier, l'existence d'une forte affinité chimique entre ces éléments peut conduire à une stabilisation des dislocations extrinsèques dans les joints généraux.

L'intérêt essentiel de cette étude est d'avoir dégagé le rôle important des ségrégations intergranulaires sur le comportement des dislocations extrinsèques des joints de grains et d'avoir souligné les conséquences que cela implique sur les propriétés des matériaux.

La différence de comportement entre joints coïncidents et non coïncidents est seulement apparente dans les métaux de haute pureté. Dans les matériaux réels, il est indispensable de tenir compte de la présence d'impuretés si l'on veut pouvoir relier les modèles théoriques aux propriétés de ces matériaux.

\section{Bibliographie}

[1] Priester, L., Revue Phys. Appl. 15 (1980) 789.

[2] Pumphrey, P. H., Gleiter, H., Philos. Mag. 30 (1974) 593.

[3] Varin, R. A., Wyrzykowski, J. W., Lojkowski, W., GrabSKI, M. W., Phys. Stat. Sol. (a) 45 (1978), 565.

[4] Varin, R. A., Phys. Status Solidi A 52 (1979) 347.

[5] Dingley, D. J., Pond, R. C., Acta Metall. 27 (1979) 667.

[6] Grimmer, H., Acta Crystallogr. Sect. A 30 (1974) 685.

[7] Bonnet, R., Acta Crystallogr. Sect. A 36 (1980) 116.

[8] Grimmer, H., Bollmann, W., Warrington, D. H., Acta Crystallogr. Sect. A 30 (1974) 197.

[9] MykuRA, H. (à paraître).

[10] BollmanN, W., Crystal Defects and Crystallines Interfaces (Springer Verlag, New York) 1970.

[11] Warrington, D. H., Buffalini, P., Scr. Metall. 5 (1971) 771.

[12] Warrington, D. H., Boon, M., Acta Metall. 23 (1975) 599.

[13] Schober, T., Balluffi, R. W., Philos. Mag. 21 (1970) 109.

[14] Brandon, D. G., Acta Metall. 14 (1966) 1479.

[15] Ishida, Y., Hasagawa, T., Nagata, F., Trans. Jap. Inst. Met. 9 (1968) 504.

[16] Khalfallah, O., Bouchet, D., Priester, L., Mém. Sc. Rev. Mét. (à paraître).

[17] Orabi, G., Priester, L., Huntz, A. M., Mém. Sc. Rev. Mét. 74 (1977) 367.
[18] Bouchet, D., Priester, L., Lacombe, P., J. Microsc. Spectrosc. Electron. 1 (1976) 377.

[19] Bouchet, D., Priester, L., J. Mater. Sci. 14 (1979) 2205.

[20] VARIN, R. A., TANGRI, K., Scr. Metall. 14 (1980) 337.

[21] BALluffi, R. W., Interfacial Segregation, published by ASM, Ohio (1979) 193.

[22] Adamson, J. P., Martin, J. W., Acta Metall. 19 (1971) 1015.

[23] Jones, A. R., Howell, P. R., RalPh, B., J. Mater. Sci. 11 (1976) 1595.

[24] Buttler, E. P., Swann, P. R., Acta Metall. 24 (1976) 343.

[25] Khalfallah, O., Priester, L., Scr. Metall. 14 (1980).

[26] Varin, A., J. Mater. Sci. 14 (1979) 811.

[27] Hondros, E. D., Grain Boundary Structure and Properties, ed. by G. A. Chadwick and D. A. Smith (Academic Press, New York) 1976, p. 265.

[28] Boutin, F. R., J. Physique Colloq. 36 (1975) C 4-355.

[29] Forwood, C. T., Humble, P., Philos. Mag. 31 (1975) 1011.

[30] Forwood, C. T., Humble, P., Philos. Mag. 31 (1975) 1025.

[31] Aust, K. T., Reuter, J. W., Trans. Met. Soc. AIME 15 (1959) 119.

[32] Dimitrov, O., J. Physique Colloq. 36 (1975) C 4-319.

[33] Bauer, C. L., J. Physique Colloq. 36 (1975) C 4-367.

[34] Guyot, P., Simon, J. P., J. Physique Colloq. 36 (1975) C 4-141. 